\title{
Perceptual selectivity is task dependent: The pop-out effect poops out
}

\author{
CARL M. FRANCOLINI and HOWARD E. EGETH \\ Johns Hopkins University, Baltimore, Maryland 21218
}

\begin{abstract}
An attempt was made to determine why evidence for perceptual selectivity based on conceptual category (e.g., digits vs. letters) has been found in some experiments but not in others. Experiments using the partial-report paradigm find no partial-report superiority when the report is cued by category, whereas, in recent visual search studies, evidence for perceptual selectivity has been obtained for arrays containing a single item that was categorically different from the other items (e.g., a digit among letters). Using a search task, Experiment 1 investigated the possibility that the number of categorically different items in the arrays could be a determinant of selectivity. One, two, or three digits and a variable number of letters were presented on each trial, and subjects determined if a particular digit was present. No evidence of selectivity was obtained, even for the one-digit condition. Experiment 2 verified this result, and Experiment 3 extended the failure of selectivity to a search task in which the possible targets differed in color from the distractor items. In Experiment 4, subjects counted the number of digits or red letters in arrays in which black letters were the distractor items. The counting task was used to eliminate the requirement in our previous tasks that the subjects search for specific items. Evidence was obtained in the counting task for selectivity based on the color difference but not on the categorical difference. The color stimuli used in the counting task were essentially the same as those that did not yield any evidence of selectivity in the search task. The results suggest that task demands are an important determinant of whether or not perceptual selectivity will occur.
\end{abstract}

The impetus for the present study comes from two apparently discrepant results in the literature. Sperling (1960) presented subjects with 3 by 4 stimulus matrices in a partial report paradigm and found accuracy to be very high when either location (row) or color (red or black) were used as cues. However, for matrices that were half letters and half digits, the subjects' partial reports cued by category (digits or letters) were no more accurate than whole reports. Von Wright (1968) obtained results consistent with Sperling's findings. These studies suggest that subjects were able to selectively search arrays on the basis of location or color, but not on the basis of categorical difference.

Results of several recent studies (e.g., Egeth, Jonides, \& Wall, 1972; Gleitman \& Jonides, 1976; Jonides \& Gleitman, 1972, 1976), however, appear inconsistent with Sperling's earlier findings. The subject's task in these studies was either to detect any categorically different item (e.g., detect any digit among letters) or to determine if a particular categorically different item was present in an array (e.g., detect the presence of a " 4 " among letters). The dependent variable was mean reaction time (RT).

This research was supported in part by a grant from the National Science Foundation (BNS-76-01227) to Howard Egeth and James Pomerantz. The authors would like to thank James Pomerantz and John Jonides for helpful advice and Mary Amanda Dew for assistance in running subjects.
Items to be searched for by the subject are typically labeled "target set" and those to be ignored or occurring as background items are labeled "distractor set." When the target set and distractor set were categorically different (cf. consistently mapped; see Shiffrin \& Schneider, 1977), Egeth et al. (1972; Experiments 3 and 4) found that RT to detect a target item did not increase with the number of items in the array, i.e., the slope of the function relating RT to display size was near zero. Gleitman and Jonides (1976) obtained relatively flat RT functions (9.9-msec/item slopes) for detecting a categorically different target as compared to a $29.9-\mathrm{msec} / \mathrm{item}$ slope when searching for a target that belonged to the same category as the distractor items. These results were obtained using arrays in which elements were positioned on the circumference of an imaginary circle whose diameter subtended about $3^{\circ}$ of visual angle.

The great difference in search rates between and within categories, and in particular the fact that between-category search rates are nearly zero, suggests nearly perfect categorical selectivity. Subjects participating in these tasks typically report that the categorically different target-set item appears to "pop out" from the distractor items. Jonides and Gleitman suggest that this effect is due to partial processing of the display items into categories, in which case the categorically different item is tagged 
differently from the others and thus pops out. Thus, on one hand, it appears that subjects are not capable of perceptual selectivity using item category as a cue (Sperling, 1960; Von Wright, 1968), and on the other hand, they are (Egeth et al., 1972; Jonides \& Gleitman, 1976).

In Sperling's experiments, partial reports by color and category exemplify Broadbent's (1970) two stimulus-material classifications, "stimulus set" and "response set," respectively. Stimulus set is defined by some distinct and conspicuous physical properties that are inherent in the stimuli. Response set is characterized by the meaning that the stimulus conveys.

Recently, Keren (1976) has suggested that this distinction parallels Neisser's (1967) distinction between two cognitive processes, preattention and focal attention. Keren suggests that stimulus set material is processed principally in parallel and that response set material is processed principally in a serial manner. The distinction implied by Keren is not all or none, but continuous. The difference between red and black letters, for example, would seem to be an obvious physical one. Black letters and gray letters would still be physically different, but to a lesser degree. Finally, there may be physical differences between digits and letters, but here the difference is even more subtle and more likely a learned difference.

If Keren's hypothesis of a continuous relationship is correct, the flat display-size function obtained by Egeth et al. (1972) would suggest that in some cases a response set or well-learned distinction could equal or approximate the distinctiveness obtained from a gross, physical difference (stimulus set). Thus, two issues are dealt with in this paper. First, an attempt will be made to clarify the apparent inconsistency in the literature concerning perceptual selectivity with categorically different items. More specifically, we will attempt to differentiate some of the conditions under which perceptual selectivity occurs for arrays that contain items that differ in category. Second, we will examine some similarities and differences between a well-learned, categorical distinction and a gross physical distinction like color. Based on recent studies (Schneider \& Shiffrin, 1977; Shiffrin \& Schneider, 1977), it is apparent that the categorical difference is not a crucial aspect of this project, but is simply convenient for studying well-learned distinctions.

One possibly important difference between the experiments that demonstrated perceptual selectivity based on a categorical difference and those that did not, could be the number of items in each of the two categories. Sperling's arrays were 3 by 4 matrices of digits and letters. Of the 12 items, half were digits and half were letters. In the Egeth et al. (1972; Experiments 3 and 4) and Jonides and Gleitman (1976) studies, arrays contained (at most) a single item which was categorically different from the others.

The number of target-set items in a display could be important if the following kind of process model were assumed: An information processing system with limited capacity begins processing preattentively and in parallel. With only a partial, parallel processing of the display items, information concerning the categories of the items is determined. If only a single categorically different item is present, attention is drawn to the spatial location of the discrepant item, permitting further processing. Phenomenonally, this appears to the subject as "pop out." However, if attention were like an indivisible "beam," it could not be directed to several separated array locations at one time. When two or more categorically different items are present in an array, the attempted division of the beam would disrupt processing and the array would then be processed as if all the items were in the same category (i.e., in a serial manner). In other words, there is no longer a processing advantage for the categorically different items. Thus, "pop out" may occur only with displays that contain a single item that is categorically different from the other items. In Experiment 1 of the present paper, we examined this possibility by presenting arrays that contained a variable number of digits among letters.

\section{EXPERIMENT 1}

In the present experiment, the number of digits and the number of letters were varied independently. Targets were always digits, letters were always distractors. Before each block of trials, the experimenter specified the number of digits that would be shown on each trial of that block (one, two, or three). The number of letter distractors varied randomly from trial to trial. Before each trial, the subjects were given a specific target digit to search for. (Targets were actually present on half of the trials.) The logic of the study is as follows: Since targets were always digits, it would be reasonable strategy for subjects to restrict their search to just the digits in the display and to ignore the letters. Such a strategy could be implemented if digits could be segregated from letters at a low "preattentive" level. An index of how efficient subjects are at searching selectively through just the digits is provided by the slope of the function relating mean search RT to the number of letters in the display. More specifically, if that slope is zero, perfect selectivity is implied. If the model briefly sketched in the introduction is correct, for the singledigit conditions, mean RT ought to be independent of the number of letters displayed. However, if the two- or three-digit conditions disrupt processing, search time ought to increase as the number of letters is increased in those conditions. 


\section{Method}

Subjects. Twelve students at The Johns Hopkins University served as subjects in the experiment in partial fulfillment of an undergraduate course requirement. The subjects were tested individually for approximately $50 \mathrm{~min}$.

Stimulus materials. The stimuli were constructed using Prestype, 24-point, black Futura Demi numerals and uppercase letters mounted on cardboard cards within a $76 \times 76 \mathrm{~mm}$ white area. The stimulus cards were displayed in a conventional two-channel mirror tachistoscope at a viewing distance of $84 \mathrm{~cm}$. Luminances in both the stimulus and fixation channels were approximately $206 \mathrm{~cd} / \mathrm{m}^{2}$. The number of stimulus elements on the card varied from one to six. To maintain a constant overall visual angle, each stimulus element was located at one of eight equally spaced locations on the circumference of an imaginary circle.

When only one element was presented in an array, it could occur at any of the eight locations. When two elements were presented, they were located at diametrically opposed loci. When three elements were presented, two were diametrically opposed loci and the third was randomly positioned among the remaining loci. Similar procedures were used for trials with four, five, and six elements. The center-to-center distance between two diametrically opposed stimulus elements subtended a visual angle of $3.0^{\circ}$; the minimum visual angle between the edges of two adjacent elements was $.81^{\circ}$. The stimulus elements themselves were approximately $.4^{\circ}$ in height with a stroke width of $.068^{\circ}$.

The digits 2 through 9 were used as targets. Each of these digits and all possible array locations were used approximately equally often. All the letters of the alphabet (except $I, O, Q$, and S) were used as distractors and occurred approximately equally often. In an array with more than one digit or letter, a particular digit or letter was never repeated.

Design. A within-subjects design was used in which all 12 subjects received all three blocks of trials. The three blocks were formed by segregating arrays containing one, two, or three digits. Each array in a block contained the exact number of digits specified by the experimenter for that block, and, in addition, could contain a variable number of letters ranging from zero to a number such that the maximum array size was six elements (including both digits and letters). Thus, the number of letters (distractors) could vary from zero to five for arrays with one digit, from zero to four for arrays containing two digits, and from zero to three for arrays with three digits. The number of trials was held constant (five with the target present and five with the target absent) for each level of number of distractor items, both within and between blocks. Thus, there were 60,50 , and 40 trials for the blocks containing one, two, and three digits, respectively. Again, the correct response for half of the trials within each block was "present" and for the other half was "absent." Since each array contained one, two, or three digits, an "absent" response was appropriate when the specific target digit for that trial was not present in the array. The order of the blocks was counterbalanced across subjects, and the order of the trials within blocks was randomized for each subject. The subjects were informed when they made an error. The next array presented after an error was an unannounced practice trial which was not included in the data analysis.

Procedure. Before participating in the actual experiment, the subjects participated in a short practice session to familiarize them with the phenomenon of "pop out." Ten stimulus arrays were presented to each subject. The subjects were instructed to press the response button labeled "present" if they detected any digit and to press the button labeled "absent" if there was no digit in the array. The reason for wanting to familiarize the subjects with the pop-out effect was to suggest to naive subjects that it might be possible to make use of categorical information in a speeded search task.

The subjects were read the instructions by the experimenter for the complete experiment. Before each of the three blocks of trials was begun, the subjects were informed how many digits would be present in the arrays for that block of trials. The procedure for each trial was as follows. First, the experimenter vocally told the subject the particular digit which was to be the target of his search for that trial. Next, a warning buzzer sounded for $1 / 2 \mathrm{sec}$. The subjects were told to fixate their eyes on the central fixation cross when they heard the warning buzzer. Immediately after the warning buzzer, the fixation cross was replaced by the stimulus array, which remained in view for $200 \mathrm{msec}$. The fixation cross then reappeared with the offset of the stimulus array. The subjects pressed the "present" button with the right index finger if the target digit was present in the array or pressed the "absent" button with the left index finger if the target digit was not in the array. The instructions emphasized both speed and accuracy. Response time was measured from the onset of the stimulus array.

The subjects were given a 5-min rest between blocks and were offered chocolate bars to help them maintain their alertness for the duration of the experiment.

\section{Results and Discussion}

Mean "present" RT as a function of the number of letters in the display is shown in Figure 1 for the 1-, 2-, and 3-digit conditions. Mean "absent" RTs are shown in Figure 2.

An analysis of variance was performed on the data. The factors in the ANOVA were response type (i.e., present vs. absent), number of digits, and number of letters. ${ }^{1}$ A highly significant effect of letters $[F(3,33)=20.35, p<.001]$, indicated that the mean RT to find a particular digit increased as the number of letters in the arrays increased.

Subsequently, separate trend analyses were conducted for the "present" response of the 1-, 2-, and 3-digit conditions. The linear components for the

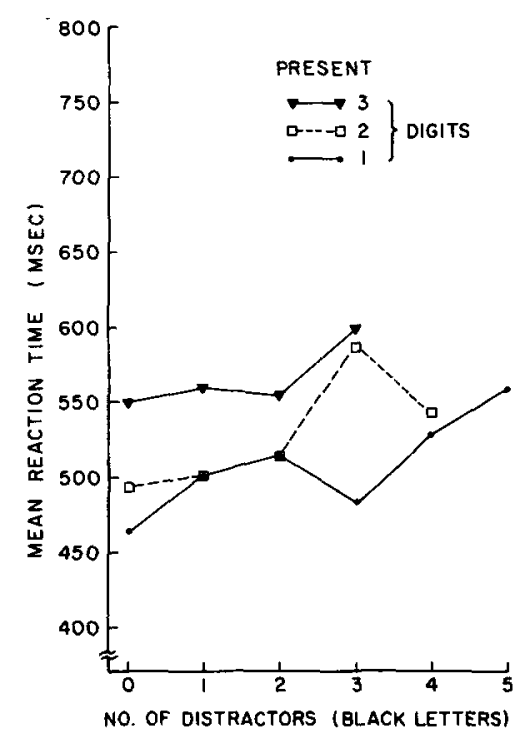

Figure 1. Mean "present" reaction time plotted as a function of the number of letter distractors for the 1-, 2-, and 3-digit conditions. Experiment 1. (To obtain the total array size for a given data point on the graph, add 1, 2, or 3-corresponding to the 1-, 2-, or 3-digit conditions-to the number of distractor items). 


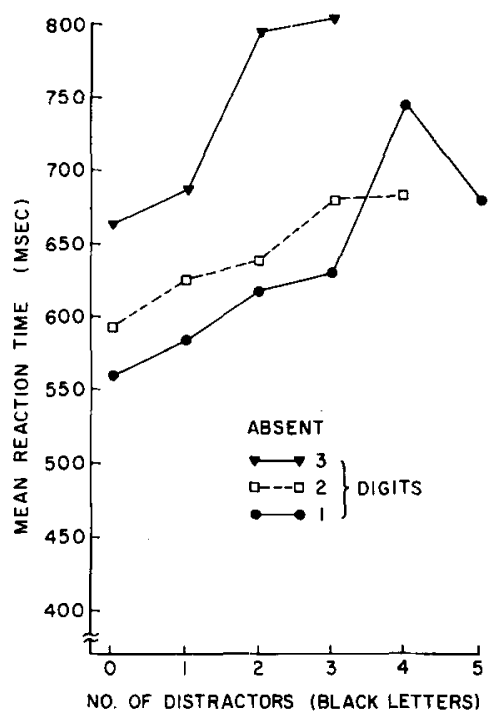

Figure 2. Mean "absent" reaction time plotted as a function of the number of letter distractors for the 1-, 2-, and 3-digit conditions. Experiment 1. (To obtain the total array size for a given data point on the graph, add 1, 2, or 3-corresponding to the 1-, 2-, or 3-digit conditions-to the number of distractor items.)

$1-, 2-$, and 3-digit conditions were all significant $[F(1,11)=11.56, p<.01 ; F(1,11)=9.76, p<.01 ;$ and $F(1,11)=4.97, \mathrm{p}<.05$, respectively]. The quadratic component was not significant for any condition.

For the "present" responses, the slopes of the functions for the 1-, 2-, and 3-digit conditions were $13.0,18.7$, and $14.1 \mathrm{msec} /$ letter, respectively. The corresponding slopes for "absent" responses were $31.7,23.7$, and $54.5 \mathrm{msec} /$ letter, respectively.

Unlike the Egeth et al. (1972) and Jonides and Gleitman (1972) experiments, nonzero slope functions were obtained, even for the 1-digit condition. Thus, the search of the arrays was not perfectly selective. The 15.2-msec/letter mean slope for the "present" responses, although not flat, is quite shallow, suggesting that perhaps subjects are able to perform a partially selective search of the arrays. In other words, it is possible that subjects perform a fast, serial search of the arrays. When the subject is searching for a particular digit, he may be able to decide more quickly that an item is not the specified target digit when it is a letter than when it is a digit. An example of a partially selective search would be the following. Suppose that, in a within-category search (i.e., looking for a " 4 " among digits), it is necessary for the exact identity of each item in the array to be determined in order to perform accurately on the task. Search would be partially selective for a between-category search task (e.g., look for a " 4 " among letters) if it were not necessary for the subject to determine the exact identity of a letter when it occurred in the search, just that it did not belong to the category of digits.

The effect of number of digits was significant $[F(2,22)=7.80, p<.005]$. On the average, adding a digit to an array increased mean RT by $53 \mathrm{msec}$. Additional letters increased mean RT by $25.8 \mathrm{msec}$ (averaged over "present" and "absent" responses). This contrast could be taken as support for the idea of a partially selective search, although one should note that this support is tenuous, since the $25.8-\mathrm{msec} /$ letter increase in RT is a within-block effect, while the 53-msec/digit increase is a between-block effect. An alternative to this partial processing hypothesis is simply that the two-stimulus materials, digits and letters, are inherently different in the ease with which subjects can search through arrays composed of each. In other words, subjects may be able to identify and search through letters more easily than digits. If a within-category search (for a letter among letters) resulted in approximately the same $15-\mathrm{msec} / \mathrm{letter}$ slope as in the between-category experiment, then we could conclude that subjects were not performing a partially selective search of the arrays, and that the search rates through digits and letters are different. (Experiment 2 suggests that this is, in fact, the case.)

The main effect of response type was highly significant $[F(1,11)=60.80, p<.001]$, indicating that the "absent" responses were significantly slower than the "present" responses. The Number of Letters by Response Type interaction was significant $[F(3,33)=6.08, p<.005]$. The $15-\mathrm{msec} /$ letter mean slope for the "present" responses is approximately one-half the mean slope for the "absent" responses (37 msec/letter). This slope difference suggests that subjects are performing a serial, selfterminating search of the arrays; i.e., subjects are able to stop searching the arrays as soon as they locate the target digit.

The only other significant effect was the Response Type by Number of Digits interaction $[F(2,22)=$ $4.70, p<.025]$. The Number of Digits by Number of Letters interaction $[F(6,66)=2.08]$ and the Response Type by Number of Digits by Number of Letters interaction $[F(6,66)=1.70]$ were not significant (p > .05). Overall mean error rates for the "present" and "absent" responses were $6.6 \%$ and $5.0 \%$, respectively. These error rates exhibited no discernible pattern across the number of letters for any digit condition.

The general conclusion that can be drawn from this experiment is that, at least for our particular experimental task, subjects were unable to perform a completely selective search of the arrays. The type of search we observed could be either a serial, selfterminating search that is partially selective, or a serial, self-terminating search with very rapid scanning and possibly different search rates for digits and 
letters. A within-category search (for a letter among letters) was given to subjects in Experiment 2 to determine the search rate through letters alone. Comparison of that search rate with those obtained in Experiment 1 enabled us to determine that the search through the letters (distractors in Experiment 1) required processing similar to that for a withincategory search.

\section{EXPERIMENT 2}

The nonflat display-size functions obtained in Experiment 1 indicated that subjects were not perfectly selective in their search for a categorically different item. However, the shallowness of the slopes suggests that a partially selective search might be occurring.

To determine if a partially selective search was occurring in Experiment 1, we would need to compare the slopes obtained in that experiment with the slope obtained for a within-category search (i.e., searching for a specific letter in a display consisting solely of letters) which obviously could not be selective in the sense in which we have used that term in Experiment 1. If the slopes obtained in Experiment 2 were very similar to those obtained in Experiment 1, one could conclude that subjects were processing the letters similarly in the two experiments and were not able to ignore the letters at all. If the slopes obtained in Experiment 2 were larger than those in Experiment 1, support would be given to the partial processing hypothesis.

If the results of Experiment 2 indicated that the search in Experiment 1 was not partially selective, then it would be necessary to try to ascertain why Egeth et al. (1972) obtained selectivity and we did not.

\section{Method}

Subjects. Twelve students at The Johns Hopkins University served as subjects in the experiment in partial fulfillment of an undergraduate course requirement. The subjects were tested individually for approximately $35 \mathrm{~min}$.

Stimulus materials. Except for the fact that these arrays contained only letters and no digits, the arrays were constructed in essentially the same way as those in Experiment 1. Each letter occurred as the target approximately equally often $(I, O, Q$, and $S$ were not used in these arrays), and the target occurred approximately equally often at all possible array locations.

Design. As in Experiment 1, a search task was used. In this experiment, however, the target items belonged to the same category as the nontargets (i.e., only letters). On each trial, the subject searched the arrays for a particular letter which was specified by the experimenter before each trial.

Ten stimulus cards were prepared for each array size (1-5), five "present" and five "absent," for a total of 50 cards. Twelve stimulus cards were also prepared to be used as practice trials before the experiment proper. All 50 stimulus cards were randomized within a single block. The stimulus arrays were shown to each subject in a different random order.
Procedure. The subjects were read the instructions by the experimenter. Each trial began with the experimenter informing the subject which particular letter was to be the target letter for that trial. In all other respects, the procedure was the same as in Experiment 1.

\section{Results and Discussion}

The "present". and "absent" data of Experiment 2 are shown in Figure 3, with mean RT plotted as a function of the number of items in the array. A twoway analysis of variance (Response Type by Number of Letters) was performed. A significant effect of response type was obtained $[F(1,11)=28.77, p<$ $.001]$, with "absent" responses slower than "present" responses. The effect of number of letters was highly significant $[F(4,44)=11.61, p<.001]$. Mean $R T$ to determine the presence of a particular letter increased as the number of letters in the arrays increased.

The slope for the "absent" responses was $39 \mathrm{msec} /$ letter. The 17-msec/letter slope for the "present" responses is again approximately one-half that of the "absent" responses. Thus, the subjects appear to be performing a serial, self-terminating search of the arrays indicated by the Response Type by Numbers of Letters interaction, which was significant $[F(4,44)$ $=3.69, \mathrm{p}<.025$ ].

The error rates for the "present" and "absent" responses were $8.9 \%$ and $5.9 \%$, respectively. These error rates exhibited no discernible trends across the number of letters for either the "present" or "absent" response conditions.

The 17- and 39-msec/letter slopes for the "present" and "absent" responses, respectively, in this experiment are in very close agreement with the 15 - and 37 msec/letter slopes obtained in Experiment 1. We can

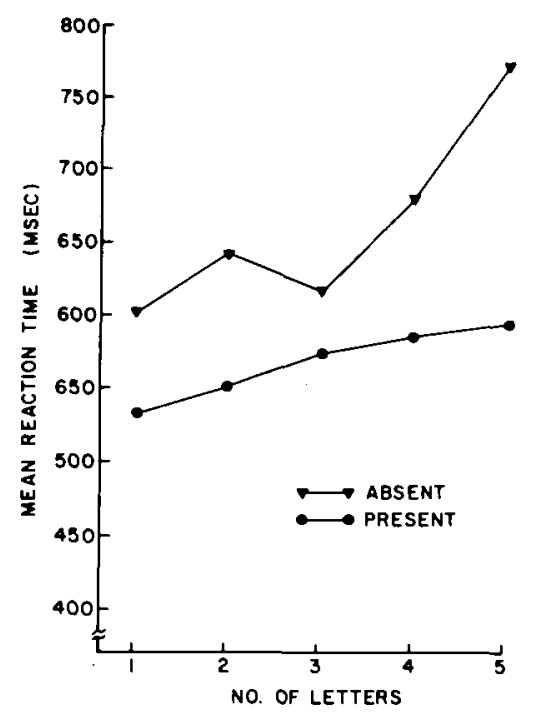

Figure 3. Mean reaction time as a function of the number of items in the display. Experiment 2. 
conclude that the subjects were probably completely processing the letters in Experiment 1 and that they were not performing even partially selective search of the arrays. (To complete the picture, another experiment ought to be conducted to determine if the search rate through digits is different from that through letters.) A tentative conclusion that may be drawn from Experiments 1 and 2 is that a categorical difference between stimuli cannot serve as the basis of a fully selective search. This is consistent with the findings of Sperling (1960) and von Wright (1968), but apparently inconsistent with the findings of Egeth, Jonides, and Wall (1972) and others. Where, then, does this leave the observation that a target may spontaneously emerge or pop out of a background of categorically different elements? Before attempting to answer this question, it may be useful to determine the extent to which search may be selective when a gross physical difference among stimuli may be used as the basis for selectivity. On the basis of Sperling's (1960) and Von Wright's (1968) data on color cues, we have assumed that if target elements were always red (and thus that black elements could never be targets), then the time required to locate a specific target would depend on the number of other red elements in the display, but not on the number of black elements. On the one hand, if such a finding obtained, it would validate our procedure and thus strengthen our finding that a categorical difference does not provide a sufficient basis for selective search. On the other hand, if the results based on a color difference were similar to those based on a categorical difference, this might indicate that our procedure (searching arrays for a specific target) is inappropriate to the study of perceptual selectivity based upon categorical differences between the display items.

\section{EXPERIMENT 3}

The purpose of this study was to determine the efficiency of selectivity based on a clear physical difference between stimuli. Subjects searched through arrays of letters for a particular target letter. They were told that if a target were present it could only be a red letter, never a black one.

\section{Method}

Subjects. Twelve students at The Johns Hopkins University served as subjects in the experiment in partial fulfillment of an undergraduate course requirement. The subjects were tested individually for approximately $55 \mathrm{~min}$.

Design. The stimulus arrays were blocked according to the number of red elements that were in each array. Since each array contained one, two, or three red letters, three blocks of trials were formed. Arrays within a block contained a variable number of black letters, ranging from zero to that number such that the maximum array size was equal to six when the red letters were included. Each subject received all three blocks and the order of the blocks was counterbalanced across subjects. Each subject received a different random order of the arrays within a block.

The stimulus arrays were made exactly as in Experiment 1, except for the following. The physically different letters were the same style and size (Futura Demibold, 24-point) but red in color. The irrelevant items (i.e., black letters) in the arrays in this experiment were exactly the same irrelevant items and in exactly the same locations as the irrelevant items used in Experiment 1. The red letters were randomly selected and assigned to the locations which were previously occupied by the digits in Experiment 1 . In an effort to obtain more stable data, six, instead of five, stimulus cards were prepared for each condition. Arrays for the additional stimulus card in each condition were randomly generated. A red letter in an array never also occurred as a black letter in the same array. A target occurred on half of the trials and if the target was present in the array, it was always a red letter, never a black one.

Procedure. Before each of the three blocks of trials was begun, the subjects were informed how many red letters would be present in each array for that block of trials. The subjects were also informed that target items would always be red, never black, and that it would be to their advantage to simply ignore the black items.

The procedure for each trial was as follows. The experimenter told the subject which particular letter was to be the target of the search for that trial. The remainder of the procedure was exactly as in Experiment 1, except that the duration of the stimulus array was changed from 200 to $175 \mathrm{msec}$.

\section{Results and Discussion}

Mean RTs for "present" and "absent" responses as a function of the number of distractors (black letters) are shown in Figure 4. An analysis of variance was performed. The factors in the ANOVA were response type, number of red letters, and number of black letters (for the $0,1,2$, and 3 levels of blackletter conditions only). ${ }^{2}$ "Absent" responses were

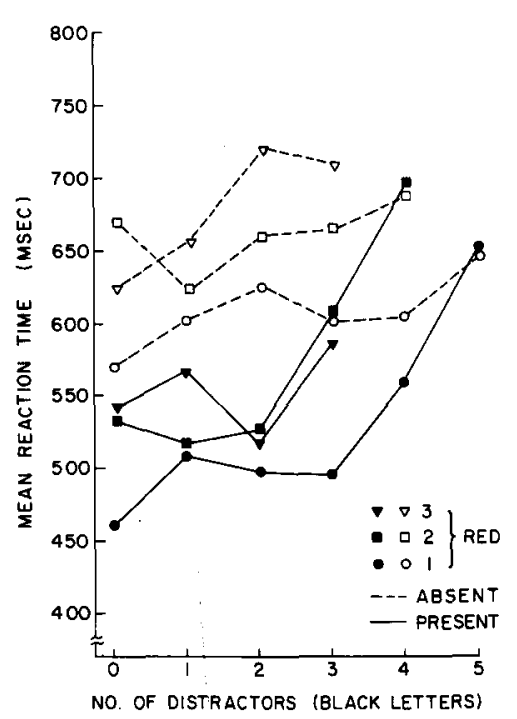

Figure 4. Mean reaction time as a function of the number of letter distractors for the 1-, 2-, and 3-red-letter conditions. Experiment 3. (To obtain the total array size for a given data point on the graph, add 1, 2, or 3-corresponding to the 1-, 2-, or 3-red-letter conditions-to the number of letter distractors.) 
114 msec slower than "present" responses $[F(1,11)$ $=18.9, \mathrm{p}<.005]$. The main effect of number of black letters was significant $[F(3,33)=7.85$, $p<$ $.001]$. This indicates that subjects were not able to attend only to the red letters and ignore the black letters. As in Experiment 1, a perfectly selective search of the arrays was not obtained. The main effect of number of red letters was significant $[F(2,22)=4.24$, $\mathrm{p}<.05$ ], indicating that mean RT increased as the number of red letters increased. The Number of Red Letters by Number of Black Letters interaction was also significant $[F(6,66)=3.48, p<.01]$. We have no particular interpretation to offer for this effect. No other effects reached significance $(p>.10)$.

The slopes for the "present" responses are 21.6, 36 , and $9.4 \mathrm{msec} /$ letter for the 1-, 2-, and 3-red-letter conditions, respectively. Trend analyses indicated significant linear components for the 1- and 2-redletter conditions $[F(1,11)=27.31, p<.001$, and $F(1,11)=14.85, p<.005$, respectively]. For the "absent" responses, the slopes are 13.2, 14.1, and $32.7 \mathrm{msec} /$ letter for the 1-, 2-, and 3-red-letter conditions, respectively.

The overall error rates for the "present" and "absent" responses were $3.5 \%$ and $2.7 \%$, respectively. These error rates showed no obvious trends across the number of black letters (distractors) for any red-letter conditions.

Except for the 3-red-letter condition, slopes were substantial and significantly greater than zero. We do not have an explanation for every detail of the results obtained in this experiment. Instead, we direct the reader's attention to the overall trends in the data. More specifically, we have no interpretation for either the "flatness" of the slope for the 3-red-letter condition in the "present" responses or for the fact that the 3-red-letter condition has the steepest slope among the "absent" responses. What seems most important, however, is that the difference between the task used in the present series of experiments and that of Egeth et al. (1972) is not a trivial one. When subjects are required to search for a particular item, even when the possible target items are distinguishable from the distractors by a gross, physical characteristic such as color, completely selective search of the arrays does not occur.

An increase in mean RT as a function of the number of colored irrelevant items has been found previously in the literature. Green and Anderson (1956) informed subjects of the color of a particular digit as a cue and found RT to locate that digit to be a function of the number of irrelevant items in the arrays. Their arrays were 60 two-digit numbers arranged in a 6 by 10 matrix $(16.75 \times 12$ in. wide $)$ presented at a distance of $10 \mathrm{ft}$. In their experiment, however, the number of irrelevant items was confounded with the number of different colors (array size was increased in increments of 15 items, and a different color was used for each additional batch of 15 items). From the results of our experiment with only a single color for the irrelevant items (black), it can be inferred that the number of items was the relevant factor and not necessarily the number of colors.

The failure to obtain selectivity for search among the red letters could have an alternative explanation other than the difficulty involved with searching for a specific target item. There is a much smaller brightness difference for a red letter on a white background than for a black letter on a white background. It could be the case that our red letters were not as "vivid" or compelling as the black letters, because of the high contrast of the black letters.

In a control study, 12 subjects were run under the same experimental conditions as the above experiment, except that the targets were always black and the distractors were red. This control study was essentially the reverse of the present study. In fact, the stimuli were made by exactly replicating the stimulus cards in the present experiment, except for changing the colors of the red letters to black and black letters to red (the same letters in the same array locations were used). The results of the control study replicated those reported here. In particular, trend analyses yielded significant linear components, indicating that mean RT increased significantly as the number of red letters (distractors, in this experiment) increased. Thus, search for a specific target was not selective among possible target letters that were a different color than the distractors, even when the possible targets were of greater contrast than the distractors.

\section{EXPERIMENT 4}

The results from Experiments 1 and 2 indicated that no pop-out or selective search of the categorically different items in the arrays was occurring. From the results obtained in Experiment 3, even with search based on a physical difference, a perfectly selective search was not obtained (nonzero slope functions were obtained). As mentioned previously, the task given to the subjects in Experiments 1 and 3 was slightly different from that used by Egeth et al. (1972). In Experiments 1-3, the subjects did not just detect the presence of a categorically different item, but had to indicate if a specific target item was present. It could be that this different or additional identification stage alters the subjects' processing mode by eliminating or disrupting the partial processing that might enable selective search of the arrays.

Experiment 4 was designed to eliminate the additional "identification stage" of processing apparently 
necessary in Experiments 1 and 3. The subject's task, in this experiment, was to count either the number of digits in arrays of digits and letters or the number of red letters in arrays of red letters and black letters. It was assumed that the identification stage would thus be eliminated, because if the categorically different items (or red letters) did pop out, it would not be necessary for the subjects to identify them-the subjects need only report the number of digits or red letters. Beck (1972) used such a counting task to assess perceptual segregation of display items that differed in various physical characteristics. Again, mean RT is plotted as a function of the number of distractors (black letters), and perfect selectivity is indicated by flat display size functions.

\section{Method}

Subjects. Eight students at The Johns Hopkins University served as subjects in the experiment in partial fulfillment of an undergraduate course requirement. The subjects were tested individually for approximately $1 \mathrm{~h}$ on 2 consecutive days.

Stimulus materials. The stimulus materials used in this experiment were randomly selected from the existing arrays used in Experiments 1, 2, and 3, or were randomly generated using the same procedures already discussed in the previous experiments.

Design. Stimulus arrays were divided into two blocks of trials. A within-subjects design was used in which all subjects received both blocks of trials. One block contained the $0-, 1-, 2-$, and 3-digit conditions and the other block the 0-, 1-, 2-, and 3-redletter conditions. For each level of the digit or red-letter conditions, the arrays contained a variable number of distractors (black letters) such that the maximum array size was six, including both digits (red letters in the corresponding block) and distractors. For example, the number of letters could vary from one to six for arrays in the 0-digit condition, from one to five for arrays with 1 digit, from one to four for arrays with 2 digits, and from one to three for arrays with 3 digits. The number of trials was held constant (seven) for each level of number of distractors, both within and between the digit (or red-letter) conditions. Thus, there were $42,35,28$, and 21 trials for the $0-, 1-, 2$-, and 3-digit conditions, respectively. The same applied for the red-letter conditions. We again wanted to avoid crowding in the arrays by maintaining the maximum array size at six items. In order to maintain the maximum array size at six items, it was necessary to choose between two alternatives. On the one hand, we could have only the 1-, 2-, and 3-black-letter conditions for each of the digit (or red-letter) conditions and thus partially confound number of digits (or red letters) with the total array size. That is, using counting red letters as an example, for a total array size of six items (including both red letters and black letters), the only possible response would be three. Alternatively, we could have allowed each individual digit (or red-letter) condition to have up to six items total. Thus, when the total array size was six, the possible responses would be "zero," "one," "two," or "three." We chose the second alternative. With this design and maintaining a constant number of trials for each black-letter condition, the probabilities for each digit (or red-letter) condition were different, but this seemed more desirable than to confound the number of digits (or red letters) with the total array size.

The order of the two blocks was counterbalanced across subjects, and each subject received the trials within a block in a different random order. The subjects received 54 unannounced practice trials at the beginning of each block.

The mode of the response in Experiment 4 was no longer a buttonpress as in the earlier experiments. Subjects now wore a headset containing a voice key and made their responses vocally.
A clock was started at the onset of the array and was stopped by the voice $k e y$. Instructions emphasized that the arrays would contain only $0,1,2$, or 3 digits or red letters, and thus the only response alternatives were "zero," "one," "two," or "three," for each trial.

Procedure. The subjects were read the instructions for the experiment. Before each of the two blocks, the subjects were informed whether they would be counting digits or red letters.

The procedure for each trial was the same as in Experiment 3 except for the following. The subjects were not looking for a particular item, but, rather, were counting the digits or red letters. Also, the duration of the stimulus arrays was changed to $1 \mathrm{sec}$. Pilot data had indicated that with $175 \mathrm{msec}$ display duration, subjects may have imposed on themselves a response-time ceiling. The array duration was lengthened in an attempt to eliminate this response ceiling (and thus reduce error rates). Any trial in which the subject slurred or stuttered was counted as an error, and the RT was not used in the data analysis.

\section{Results and Discussion}

The results for counting both red letters and digits are plotted in Figure 5. Mean RT is plotted as a function of the number of distractors (black letters). Although all data obtained from this experiment are plotted, the reported statistical analyses for all conditions are only for the one, two, and three levels of the number-of-black-letters condition, unless otherwise stated.

Separate analyses of variances were performed on the red-letter and digit data. ${ }^{3}$ The most obvious aspect of the data is that the "pop-out" effect has appeared for counting the red letters. For the redletter conditions, the main effect of number of black letters was not significant $[F(2,14)<1]$. The slopes (all levels of distractors) for the 0-, 1-, 2-, and 3-redletter conditions are $-1.6,1.7,9.3$, and $-1.5 \mathrm{msec} /$ letter, respectively. Separate trend analyses for the $0-, 1-, 2-$, and 3-red-letter conditions (all levels of distractors) showed no significant $(p>.10)$ linear components $[F(1,7)<1, F(1,7)<1, F(1,7)=3.19$,

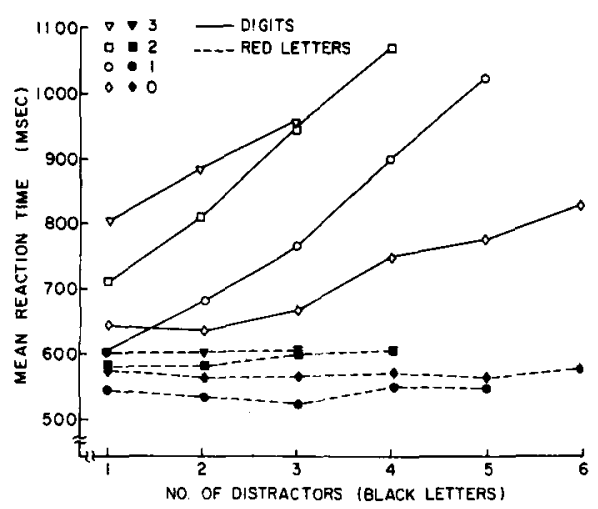

Figure 5. Mean reaction time as a function of the number of letter distractors for the $0-, 1-, 2-$, and 3-red-letter and 0-, 1-, 2-, and 3-digit conditions. Experiment 4. (To obtain the total array size for a given data point on the graph, add $0,1,2$, or $3-$ corresponding to the $0-, 1-, 2$, or 3-red-letter or digit conditionsto the number of letter distractors.) 
and $F(1,7)<1$, respectively]. A significant quadratic component was obtained only for the 1-red-letter condition $[\mathrm{F}(1,7)=6.56, \mathrm{p}<.05]$. It is obvious that RT to count the red letters is essentially independent of the number of black letters in the arrays.

The main effect of number of red letters was highly significant $[\mathrm{F}(2,14)=27.53, \mathrm{p}<.001]$. Figure 6 plots mean RT as a function of the number of red letters or digits (collapsed over number of black letters). The slope for the red-letter conditions (excluding the 0-red-letter condition) is $38 \mathrm{msec} /$ letter. Even though the red letters "popped out" from the black ones, additional red letters took additional time to count. (The lack of a significant difference between the 2- and 3-red-letter conditions may be due to the limited range for counting. In other words, since the subjects knew that 3 was the maximum number of red items, there was less uncertainty about that response and it could perhaps be speeded somewhat.) Alternatively, the increase in mean RT with additional red letters could be due to the fact that the trials for the $0-, 1-, 2-$, and 3-red-letter conditions occurred with different probabilities. Thus, subjects might have been slower on the trials with more red letters simply because fewer of these trials occurred. The number of Red Letters by Number of Black Letters interaction was not significant $(p>.05)$.

The rationale for not including the 0-red-letter condition in the overall analysis of variance is as follows. The mean RT for the 0-red-letter condition is slower than the 1-red-letter condition, but faster than the 2- and 3-red-letter conditions for all levels of distractors. It seems likely that the same type of processing that typically yields "no" responses slower than "yes" responses (perhaps double checking) would be occurring here. For this reason, it seemed inappropriate to include the 0 -red-letter condition in the analysis of variance. For the same reason and for the sake of parallel treatment, the 0-digit condi-

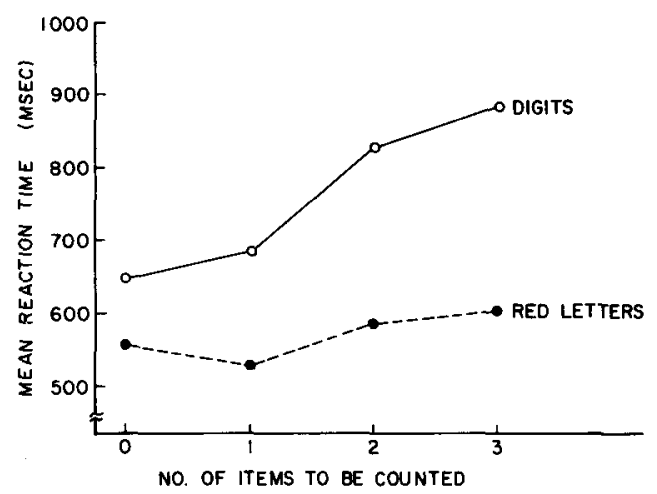

Figure 6. Mean reaction time as a function of the number of "to be counted" items (digits or red letters) collapsed over the number of letter distractors. Experiment 4. tion was not included in the analysis of variance for the digits.

For counting digits, the main effect of number of black letters was highly significant $[F(2,14)=34.68$, $\mathrm{p}<.001$ ]. Mean RT increased as the number of black letters increased. When the subjects were counting digits among letters, they were not able to ignore the letters.

The slopes (including all levels of distractors) are $40.1,107.6,120.4$, and $73.5 \mathrm{msec} /$ letter for the 0 -, 1-, 2-, and 3-digit conditions, respectively. Trend analyses (all levels of distractors) yielded significant linear components for the $0-, 1-, 2-$, and 3-digit conditions $[\mathrm{F}(1,7)=55.11, \mathrm{p}<.001 ; \mathrm{F}(1,7)=63.94$, $\mathrm{p}<.001 ; \mathrm{F}(1,7)=22.74, \mathrm{p}<.005 ;$ and $\mathrm{F}(1,7)=$ $9.86, \mathrm{p}<.025$, respectively]. A significant quadratic component was obtained only for the 0 -digit condition $[F(1,7)=6.53, p<.05]$. It is obvious that $R T$ to count the digits is not independent of the number of black letters in the arrays. Very much in contrast with the red letters, when subjects were counting, the digits did not "pop out" from the letters.

The main effect of number of digits was significant $[F(2,14)=49.62, p<.001]$. The slope for the digit conditions as plotted in Figure 6 is $98 \mathrm{msec} /$ letter (excluding the 0 -digit condition). We have no interpretation to offer for the significant Number of Digits by Number of Distractor Letters interaction $[\mathrm{F}(4,28)=2.81, \mathrm{p}<.05]$.

The overall error rate for the red-letter conditions was $.3 \%$. The overall error rate for the digit conditions was $5.4 \%$. There were no obvious trends across the letter conditions for any digit condition.

\section{GENERAL DISCUSSION}

Experiments 1 and 2 indicated that searching for a particular digit among arrays with a variable number of digits and letters did not result in selective search for any condition. In Experiment 3, we observed that complete selectivity was not obtained even with search for a particular red letter among black letters (physical difference with identification involved). It is conceivable that the color distinction was not strong enough to permit a selective search in this identification task, and that, given a much stronger color distinction (e.g., black targets and light yellow distractors), such a search would result. Finally, in Experiment 4, a counting task was employed in an attempt to eliminate the identification stage (i.e., looking for a specific item) assumed present in our previous task. The net result was perfect selectivity of search for red letters. For counting digits in Experiment 4, consistent with the results of our previous task, no evidence was obtained for perceptual selectivity based on a well-learned, categorical difference. 
One could argue that the failure to obtain perceptual selectivity for counting the digits in Experiment 4 was possibly due to the response competition for counting digits. For example, if an array contained two elements, a letter and, say, the digit " 3 ," the correct response would be "one," although "three" might compete as a response. Not as much, or perhaps no, response competition would be expected for the red-letter condition. To respond to this point, another experiment was conducted in which eight subjects counted the number of digits in an array in one block of trials and counted the number of letters in another block of trials. Arrays contained $0,1,2$, or 3 digits (letters in the corresponding block of trials) and a variable number of $\theta$ s as distractors. $\theta$ s were chosen as distractors since they appear to be similar to both letters (e.g., O, C, and B) and digits $(0,8$, and 3$)$ and, therefore, would force subjects to clearly discriminate all elements. One would predict that the slope for the digits would be significantly steeper than for the letters if response competition was occurring. This result was not obtained.

Another possible explanation of why the counting task failed to show selectivity for the digits is based on the nature of our subjects' interpretation of the focus of the task. The emphasis in the Egeth et al. (1972) and Jonides and Gleitman (1976) tasks was to detect any categorically different item-as quickly as possible. The emphasis in our counting task was to count the number of categorically different items -as quickly as possible. Pop-out could have occurred, but the subjects might have chosen to ignore it to assure being correct. This hypothesis is further supported by the extremely large slope for the 1-digit condition (107.6 msec/letter). Thus, it is possible that our counting task was not sensitive enough to observe the delicate "pop-out" phenomenon.

Throughout this series of experiments, we have attempted to maximize the opportunities to obtain evidence of perceptual selectivity with categorical differences. One significant finding of this paper is the clarification of the conditions necessary for selectivity based on a categorical difference. Perceptual selectivity was not obtained when subjects had to search for a particular categorically different item. Egeth et al. (1972) obtained selectivity when subjects merely detected the presence of a categorically different item.

Jonides and Gleitman (1976) argued that identification of a specific, categorically different item in an array was based on processing which occurred after the categorically different item was first located by partial processing. Subjects in their experiment were divided into three groups in a between-subjects design. In all three groups, the subjects were given two targets to search for on each trial. For one group of subjects (modified between-category group), the two targets were always digits. On $50 \%$ of the trials, one of the target digits was present in arrays (letters were the distractor items). For the other $50 \%$ of the trials in which neither target was present, one-half of them ( $25 \%$ of the total trials) had only letters in the array. The other half ( $25 \%$ of the total trials) had a single digit present among the letter distractors, although it was not one of the target digits. The correct response to such a digit was "absent." This task is similar to the task given our subjects in Experiment 1, since a digit had to be processed deeply enough to determine whether it was a target digit or not. Another group of subjects (betweencategory group), although given two target digits to search for on each trial (distractors were letters), could in reality perform a "detect any digit" task, since, if a digit was present in a display, it was always a target digit. A third group of subjects (withincategory group) was also given two targets to search for on each trial, but the targets and distractor items in the arrays were both letters; thus, this was a withincategory search.

The slope of the RT function for both the modified between-category group and the between-category group was approximately $10 \mathrm{msec} / \mathrm{item}$, and the slope of the within-category group was approximately $30 \mathrm{msec} /$ item. Because the modified between-category group had a higher intercept than the betweencategory group, Jonides and Gleitman argued that identification occurred after the categorically different item was located. An alternative to this explanation is that since their experiment was a betweensubjects design, the modified between-category group just happened to be slower than the betweencategory group. Furthermore, since the slopes were greater than zero and close to the slopes obtained in our Experiment 1, it is possible that subjects in both groups were completely identifying all the items and the between-category group was not just detecting the presence of a categorically different item. Thus, our hypothesis about identification could explain their failure to obtain perfect selectivity (as indicated by the $9.9-\mathrm{msec} /$ letter slope).

The difference in slope for the within- and betweencategory searches in the Jonides and Gleitman experiments can possibly be reinterpreted at two different levels. The lowest level of interpretation is an appeal to a chance difference between the two groups. That is, the subjects in the within group simply had a slower search rate than those in the between-category groups. This explanation is obviously tenuous. Another possible explanation could be the following: On each trial, two possible targets had been given to subjects in all three search conditions. In the two between-category conditions, although subjects were identifying all the array items, it would only be 
necessary for them to compare the digit (when there was one) to the two target digits. Letters in the array need not be compared to the two targets, since targets were always digits. In the within-category condition, a display item would have to be compared to both target items held in memory. In other words, there may have been some selectivity occurring in this experiment, but only in terms of memory comparisons and not perceptual selectivity. The greater number of memory comparisons could explain the steeper slope for the within-category condition. This reinterpretation of the difference in slopes for the within- and between-category obtained by Jonides and Gleitman (1976) assumes two things. First, it assumes that, in the between-category conditions, subjects have readily available to them information concerning the categories of the array items. Second, it assumes that this categorical information can be used to direct the course of memory comparisons.

One should note that the subjects in the simple between-category conditions did not even need to actually compare a digit that was present in an array to the two digits in the target set, since, if a digit was present, it was always in the target set. Subjects in the modified between-category condition, however, due to the design of the experiment, had to compare a digit found in the array to the target set in order to assure being correct. Thus, the intercept differences for the two between-category conditions could be explained either by a simple between-subjects difference or because of the additional memory comparisons required in the modified between-category task.

The results of Experiment 3 proved to be somewhat surprising. Intuitively, one might expect mean RT would be independent of the number of irrelevant items in an array when the irrelevant items were a different color from the relevant items. As we already proposed earlier, it is possible that the red-amongblack color distinction was not strong enough for this to occur. If the subject's task was to search for a particular black letter in arrays of black and yellow letters, RT might well be independent of the number of yellow letters. (However, search for a particular yellow letter in a background of black letters might well not show perfect selectivity.) The point to be noted, however, is that, even with some color distinctions, just as with conceptual category distinctions, when subjects must search for a particular item belonging to one category and an irrelevant category is present, mean RT is not necessarily independent of the irrelevant items. Thus, maybe a range of color distinctions exists that enables the search task to be completely perceptually selective (e.g., yellow letters might be functionally separable from the black letters), not at all selective, or somewhere in between. The results of Experiment 3, in addition to the above thought experiment, lends support to Keren's suggestion (as discussed in the introduction) that the two cognitive stages of preattention and focal attention are not all or none, but continuous stages.

The stimuli used in Experiment 4 (counting task) were essentially the same as those in Experiment 3 . When the subjects looked for specific red items, this color distinction was not potent enough to enable a perceptually selective search. By merely changing the task (and probably the level of processing involved), the same color distinction clearly enabled perceptual selectivity. Stimuli obviously do not completely determine the type of processing that will result.

What can be concluded about why Sperling (1960) and Von Wright (1968) did not obtain accurate partial reports with category as a cue? Probably the fact that their task involved identification of items (subjects had to report which specific letters or digits occurred in the arrays) biased their results against obtaining accurate partial reports with category cues. Also, location of the position in the matrix which each categorically different item occupied was required of their subjects (again, perhaps involving an additional or different stage of processing). This also may have reduced partial-report accuracy. Whether the number of categorically different items in the arrays was detrimental to accurate partial report (or selectivity) has yet to be determined.

\section{REFERENCES}

BECK, J. Similarity grouping and peripheral discriminability under uncertainty. American Journal of Psychology, 1972, 85, 1-19.

Brondbent, D. E. Stimulus set and response set: Two kinds of selective attention. In D. I. Mostofsky (Ed.), Attention: Contemporary theories and analysis. New York: AppletonCentury-Crofts, 1970.

Egeth, H., Jonides, J., \& Wall, S. Parallel processing of multielement displays. Cognitive Psychology, 1972, 3, 674-698.

Gleitman, H., \& Jonides, J. The cost of categorization in visual search: Incomplete processing of targets and field items. Perception \& Psychophysics, 1976, 20, 281-288.

Green, B. F., \& ANDERson, L. Color coding in a visual search task. Journal of Experimental Psychology, 1956, 51, 19-24.

Jonides, J., \& Gleitman, H. A conceptual category effect in visual search: 0 as a letter and a digit. Perception \& Psychophysics, 1972, 12, 457-460.

Jonides, J., \& Gleitman, H. The benefit of categorization in visual search. Perception \& Psychophysics, 1976, 20, 289-298.

KEREN, G. Some considerations of two alleged kinds of selective attention. Joumal of Experimental Psychology: General, 1976, 105, 349-374.

NeIsser, U. Cognitive psychology. New York: Appleton-CenturyCrofts, 1967.

SChNeIDER, W., \& Shiffrin, R. M. Controlled and automatic human information processing: I. Detection, search, and attention. Psychological Review, 1977, 84, 1-66.

ShIfFrin, R. M., \& Schneider, W. Controlled and automatic human information processing: II. Perceptual learning, automatic attending, and general theory. Psychological Review, 1977, 84, 127-190.

SPERLING, G. The information available in brief visual presentations. Psychological Monographs: General and Applied, 1960. 74(11, Whole No. 498).

Von Wright, J. M. Selection in visual immediate memory. Quarterly Journal of Experimental Psychology, 1968, 20, 62-68. 


\section{NOTES}

1. Because the maximum array size was six items (including both digits and letters) for the 1-, 2-, and 3-digit conditions, there were six levels of letters for the 1-digit condition (zero to five letters), five levels for the 2-digit condition (zero to four letters), and four levels for the 3-digit condition (zero to three letters). The number-of-letters factor in the three-way ANOVA used only the 0-, 1-, 2-, and 3-letter conditions (which were present in all digit conditions). However, the subsequent trend analyses and reported slopes are based on all of the data in a given digit condition.

2. Since the design of this experiment was the same as in Experiment 1, there were six levels of black letters (zero through five) for the 1-red-letter condition, five levels (zero to four) of black letters for the 2-red-letter condition, and four levels (zero to three) of black letters for the 3-red-letter condition. Again, the overall analysis of variance used only the $0-, 1-, 2-$, and 3-black-letter conditions for the number-of-black-letters factor. Subsequent trend analyses and reported slopes are based on all levels of distractors for each red-letter condition.

3. Each two-way ANOVA was computed using only the 1-, 2-, and 3-digit (or red-letter) conditions and did not include the 0 -digit (or 0 -red-letter) condition. The reason for this will be discussed shortly in the main body of the paper.

(Received for publication July 21, 1978; revision accepted November 13, 1978.)

\section{ERRATUM}

Allan, L. G. Comments on current ratio-setting models for time perception. Perception \& Psychophysics, 1978, 24, 444-450-In Equations 13, 15, 16, and 18, (1-p) should be $(1+$ p). (The data analysis reported in Table 3 is based on the correct form of the equations.) 